\title{
D-optimal chemical balance weighing designs with diagonal covariance matrix of experimental errors
}

\author{
Małgorzata Graczyk, Bronisław Ceranka \\ Department of Mathematical and Statistical Methods, Poznań University of Life Sciences, \\ Wojska Polskiego 28, 60-638 Poznań, e-mails: malgorzata.graczyk@up.poznan.pl, \\ bronislaw.ceranka@up.poznan.pl
}

\section{SUMMARY}

In this paper, some problems related to determining experimental plans satisfying the criterion of D-optimality are presented. Moreover, the optimality conditions and relations between the parameters of the chemical balance weighing designs are described, and some construction examples are given.

Key words: agricultural experiment, chemical balance weighing design, diagonal covariance matrix, D-optimality

\section{Introduction}

We consider an experiment in which the observations can be described as linear combinations of unknown measurements of objects with factors equal to $-1,0$ or 1. Such an experiment corresponds to the following model of the chemical balance weighing design:

$$
\mathbf{y}=\mathbf{X w}+\mathbf{e},
$$

where $\mathbf{y}$ is an $n \times 1$ random vector of observations, $\mathbf{X} \in \mathbf{\Phi}_{n \times p}\{-1,0,1\}$, the class of $n \times p$ matrices with elements equal to $-1,0$ or $1, \mathbf{w}$ is a $p \times 1$ vector of uknown measurements of objects, and $\mathbf{e}$ is an $n \times 1$ random vector of errors. We assume that there are no systematic errors, i.e. $\mathrm{E}(\mathbf{e})=\mathbf{0}_{n}$, that the errors are uncorrelated and they may have different variances, i.e. $\operatorname{Var}(\mathbf{e})=\sigma^{2} \mathbf{G}$, where $\mathbf{0}_{n}$ is the $n \times 1$ vector with zero elements everywhere, and $\mathbf{G}$ is a known $n \times n$ positive definite 
diagonal matrix which describes the conditions in which the experiment was performed.

For the estimation of $\mathbf{w}$, the least squares method is applied. We use normal equations of the form $\mathbf{X}^{\prime} \mathbf{G}^{-1} \mathbf{X} \widehat{\mathbf{w}}=\mathbf{X}^{\prime} \mathbf{G}^{-1} \mathbf{y}$, and under the assumption that $\mathbf{X}$ is of full column rank, we obtain the least squares estimator of $\mathbf{w}$ in the form $\widehat{\mathbf{w}}=\mathbf{M}^{-1} \mathbf{X}^{\prime} \mathbf{G}^{-1} \mathbf{y}$ and the covariance matrix of $\widehat{\mathbf{w}}$ given as $\operatorname{Var}(\widehat{\mathbf{w}})=\sigma^{2} \mathbf{M}^{-1}$, where $\mathbf{M}=\mathbf{X}^{\prime} \mathbf{G}^{-1} \mathbf{X}$ is called the information matrix for the design $\mathbf{X}$.

Problems regarding optimality criteria for chemical balance weighing designs have been discussed in the literature; see Jacroux et al. (1983), Sathe and Shenoy (1990). Moreover, some construction methods of the optimal design matrix were given in papers by Ceranka and Graczyk (2014a,b, 2015, 2017). Besides, in Cheng $(1980,2014)$ the relations between chemical balance weighing designs and factorial designs are considered.

Among many optimality criteria, D-optimality is considered. The design $\mathbf{X}_{D}$ is D-optimal for the covariance matrix of errors $\sigma^{2} \mathbf{G}$ in the class $\boldsymbol{\Phi}_{n \times p}\{-1,0,1\}$ if $\operatorname{det}\left(\mathbf{X}_{D}^{\prime} \mathbf{G}^{-1} \mathbf{X}_{D}\right)=\max \left(\operatorname{det}(\mathbf{M}): \mathbf{X} \in \mathbf{\Phi}_{n \times p}\{-1,0,1\}\right)$. If $\operatorname{det}(\mathbf{M})$ attains the upper bound then the design is called regular D-optimal. In other cases, such a design is called D-optimal. Problems related to determining D-optimal chemical balance weighing designs are presented in Masaro and Wong (2008), Neubauer and Pace (2010), Katulska and Smaga (2013), Smaga (2014). Varied construction methods for different optimality criteria are presented in the literature; see for example Ceranka and Graczyk (2014a,b).

The aim of this work is to determine a D-optimal design in the class $\boldsymbol{\Phi}_{n \times p}\{-1,0,1\}$, in which it is not possible to construct such a matrix based on the known methods so far presented in the literature. We consider a chemical balance weighing design under the basic assumption that $\sigma^{2} \mathbf{G}$ is a diagonal matrix. The idea is to take a regular D-optimal design and add one or more measurements in order to obtain a D-optimal design in the class $\boldsymbol{\Phi}_{n \times p}\{-1,0,1\}$. A similar problem was presented in Ceranka and Graczyk (2017) under the assumption $\mathbf{G}=\mathbf{I}_{n}$, where $\mathbf{I}_{n}$ denotes the identity matrix. 
A similar construction problem was first considered in the case of spring balance weighing designs in Katulska and Przybył (2007).

Now, we recall the definition of a D-optimal design and the theorem determining the parameters of a regular D-optimal design given in Ceranka and Graczyk (2014a).

Definition 1.1. (Ceranka and Graczyk, 2014a) Any chemical balance weighing design $\mathbf{X} \in \boldsymbol{\Phi}_{n \times p}\{-1,0,1\}$ with the covariance matrix of errors $\sigma^{2} \mathbf{I}_{n}$ is regular D-optimal if $\operatorname{det}(\mathbf{M})=m^{p}$, where $m$ is the maximal number of elements equal to -1 and 1 in columns of $\mathbf{X}$.

Theorem 1.1. (Ceranka and Graczyk, 2014a) Any chemical balance weighing design $\mathbf{X} \in \boldsymbol{\Phi}_{n \times p}\{-1,0,1\}$ with the covariance matrix of errors $\sigma^{2} \mathbf{I}_{n}$ is regular D-optimal if and only if $\mathbf{X}^{\prime} \mathbf{X}=m \mathbf{I}_{p}$.

In the second section, the conditions determining measurements which should be added to obtain a D-optimal design are presented. Construction examples are also given in this section. The last section contains examples of applications for the considered plans of experiments.

\section{The main result}

Let $\mathbf{X}_{1}$ be the design matrix of a regular D-optimal chemical balance weighing design. The problem is to add measurements in such a way that the constructed matrix is also regular D-optimal. Additional measurements are added with different variances. From the practical point of view, such additional measurements are taken in different conditions.

\subsection{Addition of one measurement}

We consider the following covariance matrix of errors:

$$
\sigma^{2} \mathbf{G}=\sigma^{2}\left[\begin{array}{cc}
\mathbf{I}_{n-1} & \mathbf{0}_{n-1} \\
\mathbf{0}_{n-1}^{\prime} & g_{1}^{-1}
\end{array}\right], g_{1}>0
$$


Let $\mathbf{X}_{1} \in \mathbf{\Phi}_{(n-1) \times p}\{-1,0,1\}$ be the design matrix of a regular D-optimal chemical balance weighing design, i.e. $\mathbf{X}_{1}^{\prime} \mathbf{X}_{1}=m \mathbf{I}_{p}$. Now, let us consider the design $\mathbf{X} \in \boldsymbol{\Phi}_{n \times p}\{-1,0,1\}$ in the form

$$
\mathbf{X}=\left[\begin{array}{l}
\mathbf{X}_{1} \\
\mathbf{x}_{1}^{\prime}
\end{array}\right]
$$

where $\mathbf{x}_{1}$ is any $p \times 1$ vector of elements $-1,0$ or $1, \mathbf{x}_{1}^{\prime} \mathbf{x}_{1}=t_{1}, 1 \leq t_{1} \leq p$. Now, we study the function $\operatorname{det}(\mathbf{M})$. According to Theorem 18.1.1 in Harville (1997), for $\mathbf{X}$ in the form (2.2), we have $\operatorname{det}(\mathbf{M})=\operatorname{det}\left(\mathbf{X}_{1}^{\prime} \mathbf{X}_{1}\right)\left(1+g_{1} \mathbf{x}_{1}^{\prime}\left(\mathbf{X}_{1}^{\prime} \mathbf{X}_{1}\right)^{-1} \mathbf{x}_{1}\right)$. We are interested in determining the maximum of $\operatorname{det}(\mathbf{M})$ under the given matrix $\mathbf{X}_{1} \in \mathbf{\Phi}_{(n-1) \times p}\{-1,0,1\}$. We know that the function $\operatorname{det}(\mathbf{M})=m^{p-1}(m+$ $\left.g_{1} t_{1}\right)$ as a function of $t_{1}$ attains a maximum if and only if $t_{1}=p$. So we may formulate the following theorem.

Theorem 2.1. Any chemical balance weighing design $\mathbf{X}$ as given in (2.2) with the covariance matrix of errors given in (2.1) is regular D-optimal in the class $\boldsymbol{\Phi}_{n \times p}\{-1,0,1\}$ if and only if $\mathbf{x}_{1}^{\prime} \mathbf{x}_{1}=p$.

Definition 2.1. Any chemical balance weighing design $\mathbf{X}$ as given in (2.2) with the covariance matrix of errors given in (2.1) is regular D-optimal in the class $\boldsymbol{\Phi}_{n \times p}\{-1,0,1\}$ if $\operatorname{det}(\mathbf{M})=m^{p-1}\left(m+g_{1} p\right)$.

For the regular D-optimal chemical balance weighing design $\mathbf{X}$ given in (2.2) with the covariance matrix of errors given in (2.1), the matrix $\mathbf{M}$ is equal to

$$
\mathbf{M}=m \mathbf{I}_{p}+g_{1} \mathbf{x}_{1} \mathbf{x}_{1}^{\prime} \text { and } \mathbf{M}^{-1}=\frac{1}{m}\left(\mathbf{I}_{p}-\frac{g_{1}}{m+p g_{1}} \mathbf{x}_{1} \mathbf{x}_{1}^{\prime}\right) \text {. }
$$

Then

$$
\operatorname{Var}\left(\widehat{w}_{J}\right)=\frac{m+(p-1) g_{1}}{m\left(m+p g_{1}\right)} \sigma^{2}, j=1,2, \ldots, p .
$$

Example 2.1. We determine unknown measurements of $p=5$ objects in $n=7$ measurements according to the D-optimality criterion. Thus, we consider the regular D-optimal chemical balance weighing design $\mathbf{X}_{1} \in \mathbf{\Phi}_{6 \times 5}\{-1,0,1\}$ given in the form 


$$
\mathbf{X}_{1}=\left[\begin{array}{rrrrr}
-1 & -1 & 1 & 1 & 0 \\
1 & -1 & -1 & 0 & 1 \\
-1 & 1 & 0 & -1 & 1 \\
1 & 0 & 1 & -1 & -1 \\
0 & 1 & -1 & 1 & -1 \\
1 & 1 & 1 & 1 & 1
\end{array}\right]
$$

Hence, $\mathbf{X}=\left[\begin{array}{ll}\mathbf{X}_{1}^{\prime} & \mathbf{1}_{5}\end{array}\right]^{\prime} \in \mathbf{\Phi}_{7 \times 5}\{-1,0,1\}$ is a regular D-optimal design. Each of the unknown measurements of objects is determined with variance

$$
\operatorname{Var}\left(\widehat{W}_{J}\right)=\frac{5+4 g_{1}}{25\left(g_{1}+1\right)} \sigma^{2}, j=1,2, \ldots, 5 \text {. }
$$

\subsection{Addition of two measurements}

We consider the covariance matrix of errors in the form

$$
\sigma^{2} \mathbf{G}=\sigma^{2}\left[\begin{array}{ccc}
\mathbf{I}_{n-2} & \mathbf{0}_{n-2} & \mathbf{0}_{n-2} \\
\mathbf{0}_{n-2}^{\prime} & g_{1}^{-1} & 0 \\
\mathbf{0}_{n-2}^{\prime} & 0 & g_{2}^{-1}
\end{array}\right], g_{1}, g_{2}>0 .
$$

Let $\mathbf{X}_{1} \in \boldsymbol{\Phi}_{(n-2) \times p}\{-1,0,1\}$ be the design matrix of a regular D-optimal chemical balance weighing design. Now, let us consider the design $\mathbf{X} \in \boldsymbol{\Phi}_{n \times p}\{-1,0,1\}$ in the form

$$
\mathbf{X}=\left[\begin{array}{l}
\mathbf{X}_{1} \\
\mathbf{x}_{1}^{\prime} \\
\mathbf{x}_{2}^{\prime}
\end{array}\right]
$$

where $\mathbf{x}_{1}, \mathbf{x}_{2}$ are $p \times 1$ vectors of elements $-1,0$ or $1, \mathbf{x}_{s}^{\prime} \mathbf{x}_{s}=t_{s}, 1 \leq t_{s} \leq p$, $\mathbf{x}_{s}^{\prime} \mathbf{x}_{q}=\mathbf{x}_{q}^{\prime} \mathbf{x}_{s}=u_{s q}, 0 \leq u_{s q} \leq p, s, q=1,2$. We determine the maximum of the function $\operatorname{det}(\mathbf{M})$ under the given matrix $\mathbf{X}_{1}$. Using the equality given in Theorem 18.1.1 in Harville (1997) for $\mathbf{X}$ in the form (2.4) and the covariance matrix of errors in (2.3), we obtain

$$
\operatorname{det}(\mathbf{M})=\operatorname{det}\left(\mathbf{X}_{1}^{\prime} \mathbf{X}_{1}\right) \operatorname{det}\left(\mathbf{I}_{2}+\left[\begin{array}{l}
g_{1} \mathbf{x}_{1}^{\prime} \\
g_{2} \mathbf{x}_{2}^{\prime}
\end{array}\right]\left(\mathbf{X}_{1}^{\prime} \mathbf{X}_{1}\right)^{-1}\left[\begin{array}{ll}
\mathbf{x}_{1} & \mathbf{x}_{2}
\end{array}\right]\right) .
$$

We are interested in determining the maximum of $\operatorname{det}(\mathbf{M})$ under the given matrix $\mathbf{X}_{1} \in \boldsymbol{\Phi}_{(n-2) \times p}\{-1,0,1\}$. The function

$$
\operatorname{det}(\mathbf{M})=m^{p-2}\left[\left(m+g_{1} t_{1}\right)\left(m+g_{2} t_{2}\right)-g_{1} g_{2} u_{12}^{2}\right]
$$


as a function of $t_{1}$ and $t_{2}$ attains a maximum if and only if $\left(m+g_{1} t_{1}\right)$, $\left(m+g_{2} t_{2}\right)$ are maximal and $u_{12}^{2}$ is minimal. Then $t_{1}=t_{2}=p$. In this case $\operatorname{det}(\mathbf{M})=m^{p-2}\left[\left(m+g_{1} p\right)\left(m+g_{2} p\right)-g_{1} g_{2} u_{12}^{2}\right]$. Let us note that $u_{12}$ is the scalar product of two rows of the matrix having elements $-1,0,1$. So, if $p$ is even, the smallest value of $u_{12}$ is $u_{12}=0$. Then, $\operatorname{det}(\mathbf{M})=m^{p-2}\left(m+g_{1} p\right)(m+$ $g_{2} p$ ). If $p$ is odd, then the smallest value of $u_{12}$ is $\left|u_{12}\right|=1$. In this case $\operatorname{det}(\mathbf{M})=m^{p-2}\left[\left(m+g_{1} p\right)\left(m+g_{2} p\right)-g_{1} g_{2}\right]$. Hence we may formulate the following theorem.

Theorem 2.2. Any chemical balance weighing design $\mathbf{X}$ as given in (2.4) with the covariance matrix of errors given in (2.3) is regular D-optimal in the class $\boldsymbol{\Phi}_{n \times p}\{-1,0,1\}$ if and only if

(i) $t_{1}=t_{2}=p$

(ii) $\left|u_{12}\right|= \begin{cases}0 & \text { if } p \text { is even } \\ 1 & \text { if } p \text { is odd }\end{cases}$

Definition 2.2. Any chemical balance weighing design $\mathbf{X}$ as given in (2.4) with the covariance matrix of errors given in (2.3) is regular D-optimal in the class $\boldsymbol{\Phi}_{n \times p}\{-1,0,1\}$ if

$$
\operatorname{det}(\mathbf{M})=\left\{\begin{array}{cl}
m^{p-2}\left(m+g_{1} p\right)\left(m+g_{2} p\right) & \text { if } p \text { is even } \\
m^{p-2}\left[\left(m+g_{1} p\right)\left(m+g_{2} p\right)-g_{1} g_{2}\right] & \text { if } p \text { is odd }
\end{array}\right.
$$

Let $p$ be even. For the regular D-optimal chemical balance weighing design $\mathbf{X}$ given in (2.4) with the covariance matrix of errors given in (2.3), the matrix $\mathbf{M}$ is equal to

$$
\mathbf{M}=\left[\begin{array}{cc}
m \mathbf{I}_{\frac{p}{2}}+\left(g_{1}+g_{2}\right) \mathbf{1}_{\frac{p}{2}} \mathbf{1}_{\frac{p}{2}}^{\prime} & \left(g_{1}-g_{2}\right) \mathbf{1}_{\frac{p}{2}} \mathbf{1}_{\frac{p}{2}}^{\prime} \\
\left(g_{1}-g_{2}\right) \mathbf{1}_{\frac{p}{2}} \mathbf{1}_{\frac{p}{2}}^{\prime} & m \mathbf{I}_{\frac{p}{2}}+\left(g_{1}+g_{2}\right) \mathbf{1}_{\frac{p}{2}} \mathbf{1}_{\frac{p}{2}}^{\prime}
\end{array}\right] \text { and } \mathbf{M}^{-1}=\left[\begin{array}{cc}
\mathbf{F} & \mathbf{H} \\
\mathbf{H} & \mathbf{F}
\end{array}\right],
$$

where

$$
\mathbf{F}=\frac{1}{m}\left(\mathbf{I} \frac{p}{2}-\frac{m\left(g_{1}+g_{2}\right)+2 p g_{1} g_{2}}{m^{2}+p m\left(g_{1}+g_{2}\right)+p^{2} g_{1} g_{2}} \mathbf{1} \frac{p}{2} \mathbf{1}_{\frac{p}{2}}^{\prime}\right),
$$




$$
\mathbf{H}=-\frac{g_{1}-g_{2}}{m^{2}+p m\left(g_{1}+g_{2}\right)+p^{2} g_{1} g_{2}} \mathbf{1}_{\underline{2}} \mathbf{1}_{\underline{\underline{p}}}^{\prime}
$$

Then

$$
\operatorname{Var}\left(\widehat{w_{J}}\right)=\frac{m^{2}+m(p-1)\left(g_{1}+g_{2}\right)+p(p-2) g_{1} g_{2}}{m\left(m^{2}+p m\left(g_{1}+g_{2}\right)+p^{2} g_{1} g_{2}\right)} \sigma^{2}, j=1,2, \ldots, p .
$$

Let $p$ be odd. For the regular D-optimal chemical balance weighing design $\mathbf{X}$ given in (2.4) with the covariance matrix of errors given in (2.3), the matrix $\mathbf{M}$ is equal to

$$
\begin{aligned}
& \mathbf{M}=\left[\begin{array}{cc}
m \frac{\mathbf{I}_{p+1}}{2}+\left(g_{1}+g_{2}\right) \mathbf{1}_{\frac{p+1}{2}} \mathbf{1}_{\frac{p+1}{2}}^{\prime} & \left(g_{1}-g_{2}\right) \frac{\mathbf{1}_{\frac{p+1}{2}}}{\mathbf{1}_{\frac{p-1}{2}}^{\prime}} \\
\left(g_{1}-g_{2}\right) \mathbf{1}_{\frac{p-1}{2}} \frac{\mathbf{1}_{\frac{p+1}{2}}^{\prime}}{m \mathbf{I}_{\frac{p+1}{2}}}+\left(g_{1}+g_{2}\right) \mathbf{1}_{\frac{p-1}{2}} \mathbf{1}_{\frac{p-1}{2}}^{\prime}
\end{array}\right] \\
& \text { and } \mathbf{M}^{-1}=\left[\begin{array}{cc}
\mathbf{F} & \mathbf{H} \\
\mathbf{H}^{\prime} & \mathbf{K}
\end{array}\right] \text {, where } \\
& \mathbf{F}=\frac{1}{m}\left(\frac{\mathbf{I} \frac{p+1}{2}}{m^{2}+p m\left(g_{1}+g_{2}\right)+\left(p^{2}-1\right) g_{1} g_{2}} \mathbf{1}_{\frac{p+1}{2}} \mathbf{1}_{\frac{p+1}{2}}^{\prime}\right), \\
& \mathbf{H}=\frac{g_{1}-g_{2}}{m^{2}+p m\left(g_{1}+g_{2}\right)+\left(p^{2}-1\right) g_{1} g_{2}} \mathbf{1}_{\frac{p+1}{2}} \mathbf{1}_{\frac{p-1}{2}}^{\prime}, \\
& \mathbf{K}=\frac{1}{m}\left(\mathbf{I} \frac{p-1}{2}-\frac{m\left(g_{1}+g_{2}\right)+2(p+1) g_{1} g_{2}}{m^{2}+p m\left(g_{1}+g_{2}\right)+\left(p^{2}-1\right) g_{1} g_{2}} \mathbf{1}_{\frac{p-1}{2}} \mathbf{1}_{\frac{p-1}{2}}^{\prime}\right) \text {. }
\end{aligned}
$$

Then

$$
\begin{aligned}
& \operatorname{Var}\left(\widehat{W_{J}}\right)=\frac{m^{2}+m(p-1)\left(g_{1}+g_{2}\right)+(p-1)^{2} g_{1} g_{2}}{m\left(m^{2}+p m\left(g_{1}+g_{2}\right)+\left(p^{2}-1\right) g_{1} g_{2}\right)} \sigma^{2}, j=1,2, \ldots, \frac{p+1}{2} \\
& \quad \operatorname{Var}\left(\widehat{W_{J}}\right)=\frac{m^{2}+m(p-1)\left(g_{1}+g_{2}\right)+(p+1) g_{1} g_{2}}{m\left(m^{2}+p m\left(g_{1}+g_{2}\right)+\left(p^{2}-1\right) g_{1} g_{2}\right)} \sigma^{2}, j=\frac{p+3}{2}, \ldots, p .
\end{aligned}
$$

Example 2.2. We consider the case where $p$ is even. We determine unknown measurements of $p=6$ objects in $n=26$ measurements according to the D-optimality criterion. Thus, we consider the regular D-optimal chemical balance weighing design $\mathbf{X}_{1} \in \boldsymbol{\Phi}_{24 \times 6}\{-1,0,1\}$ given in the form 


$$
\mathbf{X}_{1}=\left[\begin{array}{rrrrrr}
1 & 1 & 0 & -1 & -1 & 1 \\
1 & 0 & -1 & 1 & -1 & 1 \\
0 & -1 & 1 & 1 & -1 & 1 \\
-1 & 1 & 1 & 0 & -1 & 1 \\
1 & 0 & -1 & -1 & 1 & 1 \\
1 & -1 & 1 & -1 & 0 & 1 \\
-1 & 1 & 0 & -1 & 1 & 1 \\
0 & -1 & -1 & 1 & 1 & 1 \\
-1 & 1 & -1 & 1 & 0 & 1 \\
-1 & -1 & 1 & 0 & 1 & 1 \\
-1 & -1 & 0 & 1 & 1 & -1 \\
-1 & 0 & 1 & -1 & 1 & -1 \\
0 & 1 & -1 & -1 & 1 & -1 \\
1 & -1 & -1 & 0 & 1 & -1 \\
-1 & 0 & 1 & 1 & -1 & -1 \\
-1 & 1 & -1 & 1 & 0 & -1 \\
1 & -1 & 0 & 1 & -1 & -1 \\
0 & 1 & 1 & -1 & -1 & -1 \\
1 & -1 & 1 & -1 & 0 & -1 \\
1 & 1 & -1 & 0 & -1 & -1 \\
1 & 1 & 1 & 1 & 1 & 0 \\
1 & 1 & 1 & 1 & 1 & 0 \\
-1 & -1 & -1 & -1 & -1 & 0 \\
-1 & -1 & -1 & -1 & -1 & 0
\end{array}\right] .
$$

Hence, $\mathbf{X}=\left[\begin{array}{lll}\mathbf{X}_{1}^{\prime} & \mathbf{1}_{6} & \boldsymbol{\gamma}_{1}\end{array}\right]^{\prime} \in \mathbf{\Phi}_{26 \times 6}\{-1,0,1\}$, where $\boldsymbol{\gamma}_{1}=\left[\begin{array}{lll}1 & 1 & 1-1-1-1\end{array}\right]^{\prime}$ is a regular D-optimal design. Each of the unknown measurements of objects is determined with variance

$$
\operatorname{Var}\left(\widehat{W_{J}}\right)=\frac{100+25\left(g_{1}+g_{2}\right)+6 g_{1} g_{2}}{20\left(100+30\left(g_{1}+g_{2}\right)+9 g_{1} g_{2}\right)} \sigma^{2}, j=1,2, \ldots, 6
$$

Example 2.3. We consider the case where $p$ is odd. We determine unknown measurements of $p=5$ objects in $n=12$ measurements according to the D-optimality criterion. Thus, we consider the regular D-optimal chemical balance weighing design $\mathbf{X}_{1} \in \mathbf{\Phi}_{10 \times 5}\{-1,0,1\}$ given in the form 


$$
\mathbf{X}_{1}=\left[\begin{array}{rrrrr}
0 & -1 & 1 & 1 & 1 \\
1 & 0 & -1 & 1 & 1 \\
1 & 1 & 0 & -1 & 1 \\
1 & 1 & 1 & 0 & -1 \\
-1 & 1 & 1 & 1 & 0 \\
0 & 1 & 1 & -1 & 1 \\
1 & 0 & 1 & 1 & -1 \\
-1 & 1 & 0 & 1 & 1 \\
1 & -1 & 1 & 0 & 1 \\
1 & 1 & -1 & 1 & 0
\end{array}\right]
$$

Therefore,

$$
\mathbf{X}=\left[\begin{array}{lll}
\mathbf{X}_{1}^{\prime} & \boldsymbol{\gamma}_{2} & \boldsymbol{\gamma}_{3}
\end{array}\right]^{\prime} \in \boldsymbol{\Phi}_{12 \times 5}\{-1,0,1\}
$$

where $\gamma_{2}=\left[\begin{array}{lllll}-1 & 1 & 1 & -1 & 1\end{array}\right]^{\prime}, \quad \gamma_{3}=\left[\begin{array}{lllll}1 & 1 & -1 & 1 & 1\end{array}\right]^{\prime}$ is a regular D-optimal design. Each of the unknown measurements of objects is determined with variance

$$
\begin{aligned}
& \operatorname{Var}\left(\widehat{W_{J}}\right)=\frac{4+\left(g_{1}+g_{2}\right)+g_{1} g_{2}}{4\left(8+5\left(g_{1}+g_{2}\right)+3 g_{1} g_{2}\right)} \sigma^{2}, j=1,2,3, \\
& \operatorname{Var}\left(\widehat{W_{J}}\right)=\frac{16+8\left(g_{1}+g_{2}\right)+3 g_{1} g_{2}}{16\left(8+5\left(g_{1}+g_{2}\right)+3 g_{1} g_{2}\right)} \sigma^{2}, j=4,5
\end{aligned}
$$

\subsection{Addition of three measurements}

We consider the covariance matrix of errors in the form

$$
\sigma^{2} \mathbf{G}=\sigma^{2}\left[\begin{array}{cccc}
\mathbf{I}_{n-3} & \mathbf{0}_{n-3} & \mathbf{0}_{n-3} & \mathbf{0}_{n-3} \\
\mathbf{0}_{n-3}^{\prime} & g_{1}^{-1} & 0 & 0 \\
\mathbf{0}_{n-3}^{\prime} & 0 & g_{2}^{-1} & 0 \\
\mathbf{0}_{n-3}^{\prime} & 0 & 0 & g_{3}^{-1}
\end{array}\right], g_{1}, g_{2}, g_{3}>0 .
$$

Let $\mathbf{X}_{1} \in \boldsymbol{\Phi}_{(n-3) \times p}\{-1,0,1\}$ be the design matrix of a regular D-optimal chemical balance weighing design. Now, we consider the design matrix $\mathbf{X} \in \boldsymbol{\Phi}_{n \times p}\{-1,0,1\}$ in the form

$$
\mathbf{X}=\left[\begin{array}{l}
\mathbf{X}_{1} \\
\mathbf{x}_{1}^{\prime} \\
\mathbf{x}_{2}^{\prime} \\
\mathbf{x}_{3}^{\prime}
\end{array}\right]
$$


where $\quad \mathbf{x}_{s}^{\prime} \mathbf{x}_{s}=t_{s}, \quad 1 \leq t_{s} \leq p, \quad \mathbf{x}_{s}^{\prime} \mathbf{x}_{q}=\mathbf{x}_{q}^{\prime} \mathbf{x}_{s}=u_{s q}, \quad 0 \leq u_{s q} \leq p, 1 \leq s<$ $q \leq 3, s, q=1,2,3$. We determine the maximum of the function $\operatorname{det}(\mathbf{M})$ under the given matrix $\mathbf{X}_{1}$. Using the equality given in Theorem 18.1.1 in Harville (1997), we have $\operatorname{det}(\mathbf{M})=\operatorname{det}\left(\mathbf{X}_{1}^{\prime} \mathbf{X}_{1}\right) \operatorname{det}(\mathbf{T})$, where

$$
\begin{aligned}
& \mathrm{T}=\mathbf{I}_{3}+\left[\begin{array}{l}
g_{1} \mathbf{x}_{1}^{\prime} \\
g_{2} \mathbf{x}_{2}^{\prime} \\
g_{3} \mathbf{x}_{3}^{\prime}
\end{array}\right]\left(\mathbf{X}_{1}^{\prime} \mathbf{X}_{1}\right)^{-1}\left[\begin{array}{lll}
\mathbf{x}_{1} & \mathbf{x}_{2} & \mathbf{x}_{3}
\end{array}\right] . \text { Now } \\
& \operatorname{det}(\mathbf{T})=\left(1+\frac{g_{1} t_{1}}{m}\right)\left(1+\frac{g_{2} t_{2}}{m}\right)\left(1+\frac{g_{3} t_{3}}{m}\right)+\frac{2 g_{1} g_{2} g_{3} u_{12} u_{13} u_{23}}{m^{3}}- \\
& \frac{1}{m^{2}}\left(1+\frac{g_{1} t_{1}}{m}\right) g_{2} g_{3} u_{23}^{2}-\frac{1}{m^{2}}\left(1+\frac{g_{2} t_{2}}{m}\right) g_{1} g_{3} u_{13}^{2}-\frac{1}{m^{2}}\left(1+\frac{g_{3} t_{3}}{m}\right) g_{1} g_{2} u_{12}^{2} .
\end{aligned}
$$

As we want to maximize $\operatorname{det}(\mathbf{T})$ we simultaneously determine the maximal values of

$$
1+\frac{g_{1} t_{1}}{m}, 1+\frac{g_{2} t_{2}}{m}, 1+\frac{g_{3} t_{3}}{m}
$$

and the minimal values of

$$
u_{12}^{2}, u_{23}^{2}, u_{13}^{2} \text {. }
$$

The maximum of (2.7) is attained if and only if $t_{h}=p, h=1,2,3$. Next, we consider four cases of $p$.

If $p \equiv 0(\bmod 4)$, the minimum in (2.8) is attained if and only if $u_{h s}=0$ for $1 \leq h<s \leq 3$. In this case

$$
\begin{aligned}
& \operatorname{det}(\mathbf{T}) \leq \prod_{h=1}^{3}\left(1+\frac{g_{h} p}{m}\right) \text { and } \\
& \operatorname{det}(\mathbf{M}) \leq m^{p-3} \prod_{h=1}^{3}\left(m+g_{h} p\right) .
\end{aligned}
$$

The equality in (2.9) holds if and only if $t_{h}=p, h=1,2,3$, and $u_{h s}=0$ for $1 \leq$ $h<s \leq 3$.

If $p+2 \equiv 0(\bmod 4)$, the minimum in $(2.8)$ is attained if and only if $\left|u_{h s}\right|=$ 2 or 0 , for $1 \leq h<s \leq 3,0$ appears two times and \pm 2 appears once. In this case $\operatorname{det}(\mathbf{T}) \leq \prod_{h=1}^{3}\left(1+\frac{g_{h} p}{m}\right)-4 m^{-3} A$, where $A=\min \left\{\left(m+g_{h} p\right) g_{s} g_{q}\right\}$, $h, s, q=1,2,3, h \neq s \neq q$ and then

$$
\operatorname{det}(\mathbf{M}) \leq m^{p-3}\left\{\prod_{h=1}^{3}\left(m+g_{h} p\right)-4 A\right\}
$$


The equality in (2.10) holds if and only if $t_{h}=p, h=1,2,3$, and $\left|u_{h s}\right|=2$ or 0 , for $1 \leq h<s \leq 3,0$ appears two times and \pm 2 appears once.

If $p+1 \equiv 0(\bmod 4)$ the minimal value in $(2.8)$ is attained if and only if $\left|u_{h s}\right|=1$ and $\prod_{h, s} u_{h s}=-1,1 \leq h<s \leq 3$. In this case

$$
\begin{gathered}
\operatorname{det}(\mathbf{T}) \leq \prod_{h=1}^{3}\left(1+\frac{g_{h} p}{m}\right)-\frac{2 g_{1} g_{2} g_{3}}{m^{3}}-\frac{1}{m^{2}}\left(1+\frac{g_{1} p}{m}\right) g_{2} g_{3}- \\
\frac{1}{m^{2}}\left(1+\frac{g_{2} p}{m}\right) g_{1} g_{3}-\frac{1}{m^{2}}\left(1+\frac{g_{3} p}{m}\right) g_{1} g_{2} \text { and then } \\
\operatorname{det}(\mathbf{M}) \leq m^{p-3}\left\{\prod_{h=1}^{3}\left(m+g_{h} p\right)-2 \prod_{h=1}^{3} g_{h}-\sum_{h, s, q}\left(m+g_{h} p\right) g_{s} g_{q}\right\} .
\end{gathered}
$$

The equality in (2.11) holds if and only if $t_{h}=p, h=1,2,3$, and $\left|u_{h s}\right|=1$ and $\prod_{h, s} u_{h s}=-1$.

Finally, if $p+3 \equiv 0(\bmod 4)$ the minimal value in $(2.8)$ is attained if and only if $\left|u_{h s}\right|=1$ and $\prod_{h, s} u_{h s}=1$. Then,

$$
\begin{aligned}
& \operatorname{det}(\mathbf{T}) \leq \prod_{h=1}^{3}\left(1+\frac{g_{h} p}{m}\right)+\frac{2 g_{1} g_{2} g_{3}}{m^{3}}-\frac{1}{m^{2}}\left(1+\frac{g_{1} p}{m}\right) g_{2} g_{3}-\frac{1}{m^{2}}\left(1+\frac{g_{2} p}{m}\right) g_{1} g_{3}- \\
& \frac{1}{m^{2}}\left(1+\frac{g_{3} p}{m}\right) g_{1} g_{2} \text { and } \\
& \operatorname{det}(\mathbf{M})=m^{p-3}\left\{\prod_{h=1}^{3}\left(m+g_{h} p\right)+2 \prod_{h=1}^{3} g_{h}-\sum_{h, s, q}\left(m+g_{h} p\right) g_{s} g_{q}\right\} .
\end{aligned}
$$

The equality in (2.12) holds if and only if $t_{h}=p, h=1,2,3$, and $\left|u_{h s}\right|=1$ and $\prod_{h, s} u_{h s}=1$. Thus, we may formulate the following theorem.

Theorem 2.3. Any chemical balance weighing design $\mathbf{X}$ as given in (2.6) with the covariance matrix of errors given in (2.5) is regular D-optimal in the class $\boldsymbol{\Phi}_{n \times p}\{-1,0,1\}$ if and only if

(i) $t_{1}=t_{2}=t_{3}=p$ and

(ii)

$$
\left\{\begin{array}{l}
\text { If } p \equiv 0(\bmod 4) \text { then }\left|u_{h s}\right|=0 \\
\text { If } p+2 \equiv 0(\bmod 4) \text { then }\left|u_{h s}\right|=2 \text { or } 0,0 \text { appears two times, } \pm 2 \text { appears once } \\
\text { If } p+1 \equiv 0(\bmod 4) \text { then }\left|u_{h s}\right|=1 \text { and } \prod_{h, s} u_{h s}=-1 \\
\text { If } p+3 \equiv 0(\bmod 4) \text { then }\left|u_{h s}\right|=1 \text { and } \prod_{h, s} u_{h s}=1
\end{array}\right.
$$


Definition 2.3. Any chemical balance weighing design $\mathbf{X}$ as given in (2.6) with the covariance matrix of errors given in (2.5) is regular D-optimal in the class $\boldsymbol{\Phi}_{n \times p}\{-1,0,1\}$ if $\operatorname{det}(\mathbf{M})=m^{p-3}\left\{\begin{array}{l}\boldsymbol{Z}, \text { if } p \equiv 0(\bmod 4) \\ \boldsymbol{Z}-4 A, \text { if } p+2 \equiv 0(\bmod 4) \\ \boldsymbol{Z}-2 \prod_{h=1}^{3} g_{h}-\sum_{h, s, q}\left(m+g_{h} p\right) g_{s} g_{q} \text { if } p+1 \equiv 0(\bmod 4) \\ \boldsymbol{Z}+2 \prod_{h=1}^{3} g_{h}-\sum_{h, s, q}\left(m+g_{h} p\right) g_{s} g_{q} \text { if } p+3 \equiv 0(\bmod 4)\end{array}\right.$

where $\boldsymbol{Z}=\prod_{h=1}^{3}\left(m+g_{h} p\right)$.

Example 2.4. We study the case $p \equiv 0(\bmod 4)$. We determine unknown measurements of $p=4$ objects in $n=13$ measurements according to the D-optimality criterion for $\sigma^{2} \mathbf{G}$ in (2.5) and $g_{1}=2, g_{2}=3, g_{3}=7$. Thus, we consider the regular D-optimal chemical balance weighing design $\mathbf{X}_{1} \in \mathbf{\Phi}_{10 \times 4}\{-1,0,1\}$ given in the form

$$
\mathbf{X}_{1}=\left[\begin{array}{rrrr}
0 & -1 & 1 & 1 \\
1 & 0 & -1 & 1 \\
1 & 1 & 0 & -1 \\
1 & 1 & 1 & 0 \\
-1 & 1 & 1 & 1 \\
0 & 1 & 1 & -1 \\
1 & 0 & 1 & 1 \\
-1 & 1 & 0 & 1 \\
1 & -1 & 1 & 0 \\
1 & 1 & -1 & -1
\end{array}\right]
$$

Therefore,

$$
\mathbf{X}=\left[\begin{array}{llll}
\mathbf{X}_{1}^{\prime} & \mathbf{1}_{4} & \boldsymbol{\gamma}_{4} & \boldsymbol{\gamma}_{5}
\end{array}\right]^{\prime} \in \mathbf{\Phi}_{13 \times 4}\{-1,0,1\}
$$

where $\gamma_{4}=\left[\begin{array}{llll}1 & 1 & -1 & -1\end{array}\right]^{\prime}, \gamma_{5}=\left[\begin{array}{llll}1 & -1 & -1 & 1\end{array}\right]^{\prime}$ is a regular D-optimal design and $\operatorname{Var}\left(\widehat{w}_{J}\right)=0.066 \sigma^{2}, j=1,2,3,4$.

Example 2.5. We study the case $p+2 \equiv 0(\bmod 4)$. We determine unknown measurements of $p=6$ objects in $n=9$ measurements according to the D-optimality criterion for $\sigma^{2} \mathbf{G}$ in (2.5) and $g_{1}=2, g_{2}=3, g_{3}=7$. Thus, we consider the regular D-optimal chemical balance weighing design $\mathbf{X}_{1} \in \boldsymbol{\Phi}_{6 \times 6}\{-1,0,1\}$ given in the form 


$$
\mathbf{X}_{1}=\left[\begin{array}{rrrrrr}
1 & -1 & 0 & -1 & -1 & 0 \\
0 & 1 & -1 & 0 & -1 & -1 \\
-1 & 0 & 1 & -1 & 0 & -1 \\
-1 & -1 & 0 & 1 & -1 & 0 \\
0 & -1 & -1 & 0 & 1 & -1 \\
-1 & 0 & -1 & -1 & 0 & 1
\end{array}\right]
$$

Therefore,

$$
\mathbf{X}=\left[\begin{array}{llll}
\mathbf{X}_{1}^{\prime} & \mathbf{1}_{6} & \boldsymbol{\gamma}_{6} & \boldsymbol{\gamma}_{7}
\end{array}\right]^{\prime} \in \boldsymbol{\Phi}_{9 \times 6}\{-1,0,1\}
$$

where $\gamma_{6}=\left[\begin{array}{llllll}1 & 1 & 1 & -1-1-1\end{array}\right]^{\prime}, \gamma_{7}=\left[\begin{array}{lllll}1 & 1 & -1-1-1 & -1\end{array}\right]^{\prime}$ is a regular D-optimal design. Each of the unknown measurements of objects is determined with variance $\operatorname{Var}\left(\widehat{w_{J}}\right)=0.163 \sigma^{2}, j=1,2,4,5, \operatorname{Var}\left(\widehat{w}_{J}\right)=0.117 \sigma^{2}, j=3,6$.

Example 2.6. We study the case $p+1 \equiv 0(\bmod 4)$. We determine unknown measurements of $p=3$ objects in $n=7$ measurements according to the Doptimality criterion for $\sigma^{2} \mathbf{G}$ in (2.5) and $g_{1}=2, g_{2}=3, g_{3}=7$. Thus, we consider the regular D-optimal chemical balance weighing design $\mathbf{X}_{1} \in \boldsymbol{\Phi}_{4 \times 3}\{-1,0,1\}$ given in the form

$$
\mathbf{X}_{1}=\left[\begin{array}{rrr}
0 & -1 & 1 \\
1 & 0 & -1 \\
-1 & 1 & 0 \\
1 & 1 & 1
\end{array}\right]
$$

Therefore, $\mathbf{X}=\left[\begin{array}{llll}\mathbf{X}_{1}^{\prime} & \mathbf{1}_{3} & \boldsymbol{\gamma}_{8} & \boldsymbol{\gamma}_{9}\end{array}\right]^{\prime} \in \mathbf{\Phi}_{7 \times 3}\{-1,0,1\}$, where $\boldsymbol{\gamma}_{8}=\left[\begin{array}{lll}1 & 1 & -1\end{array}\right]^{\prime}$, $\gamma_{9}=\left[\begin{array}{lll}1 & -1 & 1\end{array}\right]^{\prime}$ is a regular D-optimal design. Each of the unknown measurements of objects is determined with variance $\operatorname{Var}\left(\widehat{w_{1}}\right)=0.080 \sigma^{2}$, $\operatorname{Var}\left(\widehat{w_{2}}\right)=0.094 \sigma^{2}, \operatorname{Var}\left(\widehat{w_{3}}\right)=0.110 \sigma^{2}$.

Example 2.7. We study the case $p+3 \equiv 0(\bmod 4)$. We determine unknown measurements of $p=5$ objects in $n=13$ measurements according to the D-optimality criterion for $\sigma^{2} \mathbf{G}$ in (2.5) and $g_{1}=1.5, g_{2}=2, g_{3}=0.5$. Hence, we consider the regular D-optimal chemical balance weighing design $\mathbf{X}_{1} \in \boldsymbol{\Phi}_{10 \times 5}\{-1,0,1\}$ given in the form 


$$
\mathbf{X}_{1}=\left[\begin{array}{rrrrr}
-1 & -1 & 1 & 1 & 0 \\
-1 & 1 & -1 & 0 & 1 \\
1 & -1 & 0 & -1 & 1 \\
-1 & 0 & 1 & -1 & 1 \\
0 & 1 & 1 & -1 & -1 \\
-1 & 1 & 1 & 1 & 1 \\
1 & -1 & 1 & 1 & 1 \\
1 & 1 & -1 & 1 & 1 \\
1 & 1 & 1 & -1 & 1 \\
1 & 1 & 1 & 1 & -1
\end{array}\right]
$$

Therefore, $\quad \mathbf{X}=\left[\begin{array}{llll}\mathbf{X}_{1}^{\prime} & \mathbf{1}_{5} & \boldsymbol{\gamma}_{10} & \boldsymbol{\gamma}_{11}\end{array}\right]^{\prime} \in \boldsymbol{\Phi}_{13 \times 5}\{-1,0,1\}, \quad$ where $\quad \boldsymbol{\gamma}_{10}=$ [1 $111-1-1]^{\prime}, \boldsymbol{\gamma}_{11}=\left[\begin{array}{llll}1 & 1 & -1-1 & 1\end{array}\right]^{\prime}$ is a D-optimal design. Each of the unknown measurements of objects is determined with variance $\operatorname{Var}\left(\widehat{W}_{1}\right)=$ $0.082 \sigma^{2}, \operatorname{Var}\left(\widehat{W_{2}}\right)=0.082 \sigma^{2}, \operatorname{Var}\left(\widehat{W_{3}}\right)=\operatorname{Var}\left(\widehat{W_{4}}\right)=0.088 \sigma^{2}, \operatorname{Var}\left(\widehat{W_{5}}\right)=$ $0.085 \sigma^{2}$.

\section{Application}

Some applications of weighing designs are presented in Banerjee (1975), Harwit and Sloane (1979), Graczyk (2013), Cheng and Kao (2015). Moreover, Cheng (1980) proposed to apply the results on weighing designs to the setting of $2^{\kappa}$ fractional factorial designs. He considered a case with $\kappa$ factors, each with two levels 0 and 1 . In this setting, each design matrix consists of entries -1 and +1 , and this is the design matrix of a chemical balance weighing design. If we consider a $3^{\kappa}$ fractional factorial design, then we have $\kappa$ factors each with three levels coded by $-1,0$ or +1 . Each design matrix consists of entries $-1,0$ and +1 , and it is the design matrix of a chemical balance weighing design. The use of the chemical balance weighing design matrix as an experimental plan for factorial experiments with two or three factors seems to be useful and appropriate, as it enables estimators to be determined with minimal variance. As an example, let us consider the experiment presented in Miutu et al. (2016). The authors considered an experiment in which a three-factor factorial design was used to determine significant differences in the mean yield of maize in Nigeria with respect to the effect of fertilizers, herbicides and water volumes. The fertilizers $\{\mathrm{N}: \mathrm{P}: \mathrm{K}(20: 10: 10), \mathrm{N}: \mathrm{P}: \mathrm{K}(15: 15: 15)$, and UREA $\}$ were applied, and the maize 
was harvested on farm land and weighed per ridge in kilogram (kg). If we code the levels of these three fertilizers as $-1,0$ or +1 , then we can treat the experimental plan as the matrix of a chemical balance weighing design, and we are able to determine unknown values with the smallest variance, according to the criterion of D-optimality.

To sum up, the construction methods of experimental plans presented above are a proposal for using the design matrices of chemical balance weighing designs as experimental plans of three-factor experiments in different branches of science, especially in agricultural experiments.

\section{REFERENCES}

Banerjee K.S. (1975): Weighing Designs For Chemistry, Medicine, Economics, Operations Research, Statistics. Marcel Dekker Inc., New York.

Ceranka B., Graczyk M. (2014a): The problem of D-optimality in some experimental designs. International Journal of Mathematics and Computer Application Research 4: 11-18.

Ceranka B., Graczyk M. (2014b): Construction of the regular D-optimal weighing designs with non-negative correlated errors. Colloquium Biometricum 44: 43-56.

Ceranka B., Graczyk M. (2015): Relations between ternary designs and D-optimal weighing designs with non-negative correlated errors. Colloquium Biometricum 45: 23-34.

Ceranka B., Graczyk M. (2017): Some D-optimal chemical balance weighing designs: theory and examples. Biometrical Letters 54(2): 137-154.

Cheng C.S. (1980): Optimality of some weighing and $2^{n}$ fractional factorial designs. The Annals of Statistics 8: 436-446.

Cheng C.S. (2014): Optimal biased weighing designs and two-level main-effect plans. Journal of Statistical Theory and Practice 8: 83-99.

Cheng C.S., Kao M.H. (2015): Optimal experimental designs for fMRI via circulant biased weighing designs. The Annals of Statistics 43: 2565-2587.

Graczyk M. (2013): Some applications of weighing designs. Biometrical Letters 50(1): $15-26$.

Harville D.A. (1997): Matrix Algebra from Statistician's Perspective. Springer-Verlag, New York Inc.

Harwit M., Sloane N.J.A. (1979): Hadamard transform optics. New York, Acade-mic Press.

Jacroux M., Wong C.S., Masaro J.C. (1983). On the optimality of chemical balance weighing designs. Journal of Statistical Planning and Inference 8: 231-240.

Katulska K., Smaga Ł. (2013). A note on D-optimal chemical balance weighing designs and their applications. Colloquium Biometricum 43: 37-45. 
Katulska K., Przybył K. (2007): On certain D-optimal spring balance weighing designs. Journal of Statistical Theory and Practice 1, 393-404.

Masaro J., Wong Ch.S. (2008): D-optimal designs for correlated random vectors. Journal of Statistical Planning and Inference 138: 4093-4106.

Miutu S.O., Temitope A.O., Adekunle A.O., Joachim A.B., Olagoke A.S., Oluwaseun T.E., Oluwatoyin A. (2016): Application of Three-Factor Factorial Experimental Design with 8 Replicates per Cell: A study of Maize Yield. International Journal of Research 3(14), 620-642.

Neubauer G.N., Pace R.G. (2010): D-optimal (0,1)-weighing designs for eight objects. Linear Algebra and its Applications 432: 2634-2657.

Payne S.E. (1974): On maximizing $\operatorname{det}(\mathrm{ATA})$. Discrete Mathematics 10: $145-458$.

Sathe Y.S., Shenoy R.G. (1990): Construction method for some A- and D-optimal weighing designs when $N \equiv 0(\bmod 4)$. Journal of Statistical Planning and Inference 24: 369-375.

Smaga Ł. (2014): Necessary and sufficient conditions in the problem of D-optimal weighing designs with autocorrelated errors. Statistics and Probability Letters 92: $12-16$. 